Vorbemerkung $\ldots \ldots \ldots \ldots \ldots \ldots \ldots \ldots \ldots \ldots \ldots \ldots, \quad \mathrm{XI}$

1. Stuttgart und Tübingen (1785-1793)

1. Hegel an Haag, 8. VI. $1785 \ldots \ldots \ldots \ldots \ldots \ldots \ldots$. 3

2. Hegel an v. Rütte, 24 . VIII $1793 \ldots \ldots \ldots \ldots \ldots \ldots$

3. Hegel an v. Rütte, 11. IX. $1793 \ldots \ldots \ldots \ldots \ldots \ldots$. 5

II. Bern und Tschugg (1793-1796)

4. Stäudlin an Hegel, 14. XII. $1793 \ldots \ldots \ldots \ldots \ldots \ldots g$

5. Hölderlin an Hegel, 10./14. VII. $1794 \ldots \ldots \ldots \ldots \ldots 9$

6. Hegel an Schelling, 24. XII. $1794 \ldots \ldots \ldots \ldots \ldots \ldots$. 11

7. Schelling an Hegel, 6. I. $1795 \ldots \ldots \ldots \ldots \ldots \ldots, 13$

8. Hegel an Schelling, Ende Januar $1795 \ldots \ldots \ldots \ldots 15$

9. Hölderlin an Hegel, 26. I. $1795 \ldots \ldots \ldots \ldots \ldots \ldots \ldots 18$

10. Schelling an Hegel, 4. II. $1795 \ldots \ldots \ldots \ldots \ldots \ldots \ldots 20$

11. Hegel an Schelling, 16. IV. $1795 \ldots \ldots \ldots \ldots \ldots \ldots$. 23

12. Hegel an v. Steiger, 9. VII. $1795 \ldots \ldots \ldots \ldots \ldots \ldots .26$

13. Schelling an Hegel, 21. VII. $1795 \ldots \ldots \ldots \ldots \ldots \ldots .27$

14. Hegel an Schelling, 30. VIII. $1795 \ldots \ldots \ldots \ldots \ldots \ldots 29$

15. Hölderlin an Hegel, 25. XI. $1795 \ldots \ldots \ldots \ldots \ldots \ldots, 33$

16. Schelling an Hegel, Januar $1796 \ldots \ldots \ldots \ldots \ldots \ldots .34$

17. Schelling an Hegel, 20. VI. $1796 \ldots \ldots \ldots \ldots \ldots \ldots, 36$

18. Hegel an Hölderlin, August $1796 \ldots \ldots \ldots \ldots \ldots$. 38

19. Hölderlin an Hegel, 24. X. $1796 \ldots \ldots \ldots \ldots \ldots \ldots$ 40

20. Hegel an Hölderlin, November $1796 \ldots \ldots \ldots \ldots \ldots \quad 42$

21. Hölderlin an Hegel, 20. XI. $1796 \ldots \ldots \ldots \ldots \ldots .44$

III. Frankfurt (1797-1800)

22. Hegel an Nanette Endel, 9. II. $1797 \ldots \ldots \ldots \ldots \ldots \quad 49$

23. Hegel an Nanette Endel, 22. III. $1797 \ldots \ldots \ldots \ldots$. 51

24. Hegel an Nanette Endel, 2./17. VII. $1797 \ldots \ldots \ldots .52$

25. Hegel an Nanette Endel, 13. XI. $1797 \ldots \ldots \ldots \ldots \ldots \quad 55$

26. Sonnenschein an Hegel, 13. XI. $1797 \ldots \ldots \ldots \ldots \ldots .57$

27. Hegel an Nanette Endel, 25. V. $1798 \ldots \ldots \ldots \ldots \ldots .57$

28. Christiane Hegel an Hegel, 15. I. $1799 \ldots \ldots \ldots \ldots \ldots$. 58

29. Hegel an Schelling, 2. XI. $1800 \ldots \ldots \ldots \ldots \ldots \ldots .58$

$$
\text { IV. Jena (1801-1807) }
$$

30. Hegel an Gebr. Ramann, 8./10. VIII. $1801 \ldots \ldots \ldots 663$

31. Hegel an :Mehmel, 26. VIII. $1801 \ldots \ldots \ldots \ldots \ldots \ldots, 63$

32. Hegel an Hufnagel, 30. XII. $1801 \ldots \ldots \ldots \ldots \ldots \ldots$ 64 


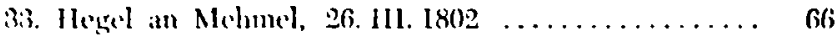

33. Seduching an Hegel, 24. V. $1802 \ldots \ldots \ldots \ldots \ldots \ldots .67$

35. Hegel an Gebr. Ramann, 25. V. $1802 \ldots \ldots \ldots \ldots \ldots 67$

36. Hegel an Gobr. Ramann, 2. VII. $1802 \ldots \ldots \ldots \ldots$ 68

37. Hufnigel an Hegel, 4. V. $1803 \ldots \ldots \ldots \ldots \ldots \ldots$ (is

i3s. Sithelling an Hegrel, 11. VII. $1803 \ldots \ldots \ldots \ldots \ldots \ldots$ 69

39. He'gel an Gocthe, 3. VIII. $1803 \ldots \ldots \ldots \ldots \ldots \ldots$ 72

40. Tregel an Shclling, 16. VIII. $1803 \ldots \ldots \ldots \ldots \ldots .72$

41. Schelling an Hegel, 31. VIII. $1803 \ldots \ldots \ldots \ldots \ldots \ldots \quad 75$

42. Hegrel an Schelling, $16 . X \mathrm{XI} 1803 \ldots \ldots \ldots \ldots \ldots \ldots .76$

43. Goethe an Hegel, 27. XI. $1803 \ldots \ldots \ldots \ldots \ldots \ldots .78$

.14. Goethe an Hegel, 15. XII. $1803 \ldots \ldots \ldots \ldots \ldots \ldots$. 79

45. Hegel an Schelling, 27. II. 1804 ............ 79

46. Schelling an Hegel, 3. III. $1804 \ldots \ldots \ldots \ldots \ldots \ldots . \quad 80$

47. Schelling an Hegel, 14, VII.1804 ............. 81

48. Hegel an Gries, 7. IX. $1804 \ldots \ldots \ldots \ldots \ldots \ldots \ldots$.

49. Hegel an Goethe, 29. IX. $1804 \ldots \ldots \ldots \ldots \ldots \ldots \ldots .84$

50. Möller an Hegel, 14. XI. $1804 \ldots \ldots \ldots \ldots \ldots \ldots \ldots .85$

51. Hegel an Goethe, 6. XII. $1804 \ldots \ldots \ldots \ldots \ldots \ldots . \quad 88$

52. Hegel an Niethammer, 10. XII. $1804 \ldots \ldots \ldots \ldots \ldots .88$

53. Niethammer an Hegel, 19. XII. $1804 \ldots \ldots \ldots \ldots \ldots .9$

54. Hegel an Niethammer, 4. III. $1805 \ldots \ldots \ldots \ldots \ldots .92$

55. Hegel an Voss, Mai $1805 \ldots \ldots \ldots \ldots \ldots \ldots \ldots .96$

56. Voss an Hegel, 24. VIII. $1805 \ldots \ldots \ldots \ldots \ldots \ldots \ldots . \ldots 2$

57. Kastner an Hegel, 15. XI. $1805 \ldots \ldots \ldots \ldots \ldots \ldots \ldots . \ldots \ldots$

58. Lange an Hegel, 4. XII. $1805 \ldots \ldots \ldots \ldots \ldots \ldots .104$

59. Hegel an Niethammer, 14. I. $1806 \ldots \ldots \ldots \ldots \ldots .105$

60. Sinclair an Hegel, 25. IV. $1806 \ldots \ldots \ldots \ldots \ldots \ldots .107$

61. Hegel an Niethammer, 17. V. $1806 \ldots \ldots \ldots \ldots \ldots .108$

62. Niethammer an Hegel, 26. V. $1806 \ldots \ldots \ldots \ldots \ldots .109$

63. Sinclair an Hegel, 27. VI. $1806 \ldots \ldots \ldots \ldots \ldots \ldots \ldots$

64. Goethe an Hegel, 27. VI. $1806 \ldots \ldots \ldots \ldots \ldots \ldots$ I1L

65. Hegel an Goethe, 30. VI. $1806 \ldots \ldots \ldots \ldots \ldots \ldots .111$

66. Sinclair an Hegel, 28. VII. $1806 \ldots \ldots \ldots \ldots \ldots \ldots 112$

67. Hegel an Niethammer, 6. VIII. $1806 \ldots \ldots \ldots \ldots \ldots .112$

68. Hegel an Niethammer, 5. IX. $1806 \ldots \ldots \ldots \ldots \ldots .114$

69. Niethammer an Hegel, 12. IX. $1806 \ldots \ldots \ldots \ldots \ldots .115$

70. Hegel an Niethammer, 17. IX. $1806 \ldots \ldots \ldots \ldots .116$

71. Niethammer 'an Hegel, 3. X. $1806 \ldots \ldots \ldots \ldots \ldots .117$

72. Hegel an Niethammer, 6. X. $1806 \ldots \ldots \ldots \ldots \ldots, 118$

73. Hegel an Niethammer, 8. X. $1806 \ldots \ldots \ldots \ldots \ldots .118$

74. Hegel an Niethammer, 13. X. $1806 \ldots \ldots \ldots \ldots .119$

75. Goethe an seine Jenaer Freunde, 18. X. $1806 \ldots \ldots, 122$

76. Hegel an Niethammer, $18 . X .1806 \ldots \ldots \ldots \ldots . . .22$ 
77. Hegel an Niethammer, 22: X. $1806 \ldots \ldots \ldots \ldots \ldots .125$

78. Hegel an Niethammer, 24. X. $1806 \ldots \ldots \ldots \ldots \ldots, 125$

79. Hegel an Niethammer, 3. XI. $1806 \ldots \ldots \ldots \ldots \ldots .126$

80. Kastner an Hegel, 15. XI. $1806 \ldots \ldots \ldots \ldots \ldots \ldots, 127$

81. Hegel an Frommann, 17. XI. $1806 \ldots \ldots \ldots \ldots \ldots .128$

82. Hegel an Schelling, 3. I. $1807 \ldots \ldots \ldots \ldots \ldots \ldots \ldots, 130$

83. Schelling an Hegel, 11. I. $1807 \ldots \ldots \ldots \ldots \ldots \ldots, 133$

84. Hegel an Niethammer, 16. I. $1807 \ldots \ldots \ldots \ldots \ldots .136$

85. Hegel an Zellmann, 23. I. $1807 \ldots \ldots \ldots \ldots \ldots \ldots . \ldots 137$

86. Schelver an Hegel, Ende Januar 1807 ........ 139

87. Hegel an Goethe, Ende Januar $1807 \ldots \ldots \ldots \ldots \ldots$ I4I

88. Niethammer an Hegel, 16. II. $1807 \ldots \ldots \ldots \ldots \ldots .143$

89. Hegel an Niethammer, 20. II. $1807 \ldots \ldots \ldots \ldots \ldots .145$

90. Hegel an Schelling, 23./27. II. $1807 \ldots \ldots \ldots \ldots \ldots .147$

\section{Bamberg (1807-1808)}

91. Sinclair an Hegel, 6. III. $1807 \ldots \ldots \ldots \ldots \ldots \ldots .155$

92. Hegel an Goethe, März $1807 \ldots \ldots \ldots \ldots \ldots \ldots \ldots .156$

93. Schelling. an Hegel, 22. III. $1807 \ldots \ldots \ldots \ldots \ldots \ldots 157$

94. Hegel an Niethammer, 7. IV. $1807 \ldots \ldots \ldots \ldots \ldots .159$

95. Hegel an Schelling, 1. V. $1807 \ldots \ldots \ldots \ldots \ldots \ldots .159$

96. Hegel an Niethammer, 2. V. $1807 \ldots \ldots \ldots \ldots \ldots, 162$

97. Sinclair an Hegel, 23. V. $1807 \ldots \ldots \ldots \ldots \ldots \ldots .163$

98. Hegel an Niethammer, 30. V. $1807 \ldots \ldots \ldots \ldots \ldots .165$

99. Hegel an Frau Niethammer, 30. V. $1807 \ldots \ldots \ldots \ldots .168$

100. Seebedk an Hegel, 29. VI. $1807 \ldots \ldots \ldots \ldots \ldots \ldots .172$

101. Hegel an Niethammer, 8. VII. $1807 \ldots \ldots \ldots \ldots .174$

102. Hegel an Niethammer, 8. VIII. $1807 \ldots \ldots \ldots \ldots .178$

103. Hegel an Niethammer, 29. VIII. $1807 \ldots \ldots \ldots \ldots .182$

104. Hegel an Knebel, 30. VIII. $1807 \ldots \ldots \ldots \ldots \ldots \ldots .185$

105. Knebel an Hegel, 11. IX. $1807 \ldots \ldots \ldots \ldots \ldots \ldots \ldots .189$

106. Hegel an Niethammer, 13. X. $1807 \ldots \ldots \ldots \ldots \ldots .191$

107. Schelling an Hegel, 2. XI. $1807 \ldots \ldots \ldots \ldots \ldots \ldots . . \ldots 4$

108. Hegel an Niethammer, November $1307 \ldots \ldots \ldots \ldots .194$

109. Hegel an Knebel, 21. XI. $1807 \ldots \ldots \ldots \ldots \ldots \ldots \ldots . \ldots 9$

110. Knebel an Hegel, 27. XI. $1807 \ldots \ldots \ldots \ldots \ldots \ldots .202$

111. Hegel an Niethammer, 23. XII. 18C' . . . . . . . 203

112. Hegel an Niethammer, 22. 1. $1808 \ldots \ldots \ldots \ldots \ldots .207$

113. Seebeck an Hegel, 29. I. 1803 .............. 211

114. Paulus und Caroline Paulus an Hegel, Jaruar 1908 .. 214

115. Caroline Paulus an IIegel, Januar $1803 \ldots \ldots \ldots \ldots 216$

116. Caroline Paulus an Hegel, Januar $1903 \ldots \ldots \ldots \ldots .217$

117. Hegel an Niethammer, 11. II. $1808 \ldots \ldots \ldots \ldots \ldots .217$

118. Seebeck an Hegel, 13. III. $1808 \ldots \ldots \ldots \ldots \ldots \ldots .219$ 
119. Hegel an Niethammer, 28. III. $1808 \ldots \ldots \ldots \ldots \ldots, 222$

120. v. Berger an Hegel; 13. IV. $1808 \ldots \ldots \ldots \ldots \ldots \ldots .223$

121. Niethammer an Hegel, 8. V. $1808 \ldots \ldots \ldots \ldots \ldots .225$

122. Hegel an Niethammer, 20. V. $1808 \ldots \ldots \ldots \ldots \ldots .226$

123. Creuzer an Hegel, 29. V. $1808 \ldots \ldots \ldots \ldots \ldots \ldots .233$

124. Hegel an Creuzer, 28. VI. $1808 \ldots \ldots \ldots \ldots \ldots \ldots .234$

125. Hegel an Frommann, 9. VII. $1808 \ldots \ldots \ldots \ldots \ldots \ldots 235$

126. Hegel an Niethammer, 20./23. VIII. $1808 \ldots \ldots \ldots \ldots .237$

127. Hegel an Niethammer, 15. IX. $1808 \ldots \ldots \ldots \ldots \ldots .240$

128. Knebel an Hegel, 28. IX. $1808 \ldots \ldots \ldots \ldots \ldots \ldots \ldots .243$

129. Hegel an Niethammer, 1. X. $1808 \ldots \ldots \ldots \ldots \ldots \ldots, 244$

130. Knebel an Hegel, 7. X. $1808 \ldots \ldots \ldots \ldots \ldots \ldots \ldots .245$

131. Hegel an Knebel, 14. X. $1808 \ldots \ldots \ldots \ldots \ldots \ldots \ldots .247$

132. Niethammer an Hegel, 25. X. $1808 \ldots \ldots \ldots \ldots \ldots .249$

133. Niethammer an Hegel, 26. X. $1808 \ldots \ldots \ldots \ldots \ldots .249$

134. Caroline Paulus an Hegel, Ende Oktober 1808 ..... 250

135. Hegel an Niethammer, 28./29. X. $1808 \ldots \ldots \ldots \ldots \ldots .251$

136. Hegel an Niethammer, 4. XI. $1808 \ldots \ldots \ldots \ldots \ldots$. 255

137. Hegel an das Generalkommissariat Bamberg, 9. XI. 1808256

138. Paulus an Hegel, 10. XI. 1808 .............. 258

139. Generalkommissariat Nümberg an Hegel, 15. XI. 1808259

140. Niethammer an Hegel, 17. XI. $1808 \ldots \ldots \ldots \ldots \ldots .259$

141. Hegel an Niethammer, 22. XI. $1808 \ldots \ldots \ldots \ldots \ldots \ldots .260$

142. Hegel an Herzog Karl August, November $1808 \ldots \ldots .263$

143. Paulus an Hegel, 28. XI. $1808 \ldots \ldots \ldots \ldots \ldots \ldots .264$

V1. Nürnberg (1808-1812)

144. Hegel an Niethammer, 14. XII. $1808 \ldots \ldots \ldots \ldots \ldots .269$

145. Hegel an Niethammer, 12. II. $1809 \ldots \ldots \ldots \ldots \ldots . .273$

146. Hegel an Niethammer, 20. II. $1809 \ldots \ldots \ldots \ldots \ldots .278$

147. Hegel an Niethammer, 7. v. $1809 \ldots \ldots \ldots \ldots \ldots .282$

148. Hegel an Niethammer, 26./29. VI. 1809 ........ 286

149. van Ghert an Hegel, 4. VIII. $1809 \ldots \ldots \ldots \ldots \ldots .290$

150. Hegel an Niethammer, 2./4./6./7. IX. $1809 \ldots \ldots \ldots .292$

151. Hegel an Niethammer, 4. X. 1809 ............ 295

152. Hegel an van Ghert, 16. XII. $1809 \ldots \ldots \ldots \ldots \ldots .298$

153. Hegel an Niethammer, 21.-18. XII. $1809 \ldots \ldots \ldots .300$

154. Hegel an Niethammer 15./16. JII. $1810 \ldots \ldots \ldots \ldots, 302$

155. Windischmann an Hegel, 27. IV. $1810 \ldots \ldots \ldots \ldots \ldots, 306$

156. Hegel an Niethammer, 11./12. V. $1810 \ldots \ldots \ldots \ldots .309$

157. Held an Hegel, 16. V. $1810 \ldots \ldots \ldots \ldots \ldots \ldots \ldots . \ldots . \ldots . \ldots$

158. Hegel an Windischmann, 27. V. $1810 \ldots \ldots \ldots \ldots .313$

159. Windischmann an Hegel, 31. V. $1810 \ldots \ldots \ldots \ldots \ldots . \ldots 315$

160. van Ghert an Hegel, 22. VI. $1810 \ldots \ldots \ldots \ldots \ldots \ldots, 316$ 
161. Hegel an Niethammer, 7. VIII. $1810 \ldots \ldots \ldots \ldots .318$

162. Sinclair an Hegel; 16. VIII. $1810 \ldots \ldots \ldots \ldots \ldots \ldots . . . \ldots 20$

163. Windischmann an Hegel, 30. VIII. $1810 \ldots \ldots \ldots \ldots .323$

164. van Ghert an Hegel, 21. IX. $1810 \ldots \ldots \ldots \ldots \ldots \ldots . . \ldots 24$

165. Hegel an Niethammer, 27. IX. $1810 \ldots \ldots \ldots \ldots \ldots 326$

166. Hegel an van Ghert, 15. X. $1810 \ldots \ldots \ldots \ldots \ldots . \ldots 328$

167. Hegel an Sinclair, Mitte Oktobér $1810 \ldots \ldots \ldots \ldots . . .331$

168. Hegel an Niethammer, 27. X. $1810 \ldots \ldots \ldots \ldots \ldots, 333$

169. Hegel an Niethammer, 3. XI. $1810 \ldots \ldots \ldots \ldots \ldots . .336$

170. Knebel an Hegel, Ende November $1810 \ldots \ldots \ldots \ldots .338$

171. Hegel an Knebel, 14. XII. $1810 \ldots \ldots \ldots \ldots \ldots \ldots .339$

172. Cároline Paulus an Hegel, 15. XII. $1810 \ldots \ldots \ldots \ldots . .341$

173. Hegel an Niethammer, 22. XII. $1810 \ldots \ldots \ldots \ldots \ldots . . \ldots 342$

174. Caroline Paulus an Hegel, 8. I. $1811 \ldots \ldots \ldots \ldots \ldots 345$

175. Niethammer an Hegel, 10, 1I. $1811 \ldots \ldots \ldots \ldots \ldots .346$

176. Hegel an Niethammer, 23. II. $1811 \ldots \ldots \ldots \ldots \ldots .348$

177. van Ghert an Hegel, 25. II. $1811 \ldots \ldots \ldots \ldots \ldots \ldots .350$

178. Hegel an seine Braut, 13. IV. $1811 \ldots \ldots \ldots \ldots \ldots . \ldots 52$

179. Sinclair an Hegel, J6. IV. $1811 \ldots \ldots \ldots \ldots \ldots \ldots . . \ldots 34$

180. Hegel an seine Braut, 17. IV. $1811 \ldots \ldots \ldots \ldots \ldots, 355$

181. Hegel an Niethammer, 18. IV. $1811 \ldots \ldots \ldots \ldots \ldots .356$

182. Hegel an Frau Frommann, 30. IV. 1811 ......... 357

183. Niethammer an Hegel, 5./7. V. $1811 \ldots \ldots \ldots \ldots \ldots .358$

184. Hegel an Frau Frommann, 18. V. $1811 \ldots \ldots \ldots \ldots .361$

185. Hegel an Niethammer, 30. V. $1811 \ldots \ldots \ldots \ldots \ldots . . .636$

186. Hegel an seine Braut, Sommer 1811 .......... 367

187. Hegel an seine Braut, Sommer 1811 .......... 369

188. Seebeck an Hegel, 13. VI. $1811 \ldots \ldots \ldots \ldots \ldots \ldots . \quad 370$

189. Hegel an Caroline Paulus, 13. VII. 1811 ......... 373

190. Hegel an Niethammer, 14. VII. $1811 \ldots \ldots \ldots \ldots .377$

191. Caroline Paulus an Hegel, 18. VII. $1811 \ldots \ldots \ldots \ldots . . .679$

192. Hegel an van Ghert, 29. VII. $1811: \ldots \ldots \ldots \ldots \ldots 381$

193. Stadtkommissariat Nürnberg an Hegel, 14. VIII. 1811382

194. Hegel an Niethammer, 16. VIII. $1811 \ldots \ldots \ldots \ldots . .382$

195. Hegel an Niethammer, 27. VIII. $1811 \ldots \ldots \ldots \ldots .383$

196. Hegel an Niethammer, 10. X. $1811 \ldots \ldots \ldots \ldots \ldots .385$

197. Hegel an Niethammer, 28. XII. $1811 \ldots \ldots \ldots \ldots \ldots, 391$

198. Hegel an Niethammer, 5. II. $1812 \ldots \ldots \ldots \ldots \ldots . .391$

199. Sinclair an Hegel, 5. II. $1812 \ldots \ldots \ldots \ldots \ldots \ldots \ldots . .394$

200. Hegel an Niethammer, 24. III. $1812 \ldots \ldots \ldots \ldots \ldots .396$

201. van Ghert an Hegel, 12. IV. $1812 \ldots \ldots \ldots \ldots \ldots . . .399$

202. Pfaff an Hegel, Sommer $1812 \ldots \ldots \ldots \ldots \ldots \ldots \ldots$ 401

203. Pfaff an Hegel, Sommer $1812 \ldots \ldots \ldots \ldots \ldots \ldots \ldots, 402$

204. Pfaff an Hegel, Sommer $1812 \ldots \ldots \ldots \ldots \ldots \ldots \ldots .405$

205. Niethammer an Hegel, 21. VI. $1812 \ldots \ldots \ldots \ldots \ldots .409$ 
206. Caroline Paulus und Paulus an Hegel, Sommer 1812 .. 411 207. Hegel an Niethammer, 19. VII. $1812 \ldots \ldots \ldots \ldots \ldots, 412$ 208. Hegel an Niethammer, 13. VIII. $1812 \ldots \ldots \ldots \ldots \ldots 44$ 209. Niethammer an Hegel, 20. IX. $1812 \ldots \ldots \ldots \ldots \ldots .415$ 210. Sinclair an Hegel, 12. X. $1812 \ldots \ldots \ldots \ldots \ldots \ldots, 416$ 211. Hegel an Niethammer, 23. X. $1812 \ldots \ldots \ldots \ldots \ldots, 418$ 212. van Ghert an Hegel, 26. X. $1812 \ldots \ldots \ldots \ldots \ldots \ldots .420$ 213. Caroline Paulus und Paulus an Hegel, 30. XI. 1812 .. 422 214. Niethammer an Hegel, 8. XII. $1812 \ldots \ldots \ldots \ldots \ldots .423$ 215. Hegel an van Ghert, 18. XII. $1812 \ldots \ldots \ldots \ldots \ldots \ldots 424$ 216. Hegel an Niethammer, 20. XII. $1812 \ldots \ldots \ldots \ldots \ldots .427$ 217. Sinclair an Hegel, 29. XII. $1812 \ldots \ldots \ldots \ldots \ldots \ldots .428$

Anmerkungen $\ldots \ldots \ldots \ldots \ldots \ldots \ldots \ldots \ldots \ldots \ldots \ldots, \quad \mathbf{4 3 1}$

Abkürzungen $\ldots \ldots \ldots \ldots \ldots \ldots \ldots \ldots \ldots \ldots \ldots \ldots \ldots \ldots$

Band II enthält die Briefe von 1813-1822

Band III enthält die Briefe von 1823-1831

Band IV enthält Nachträge, Dokumente, Personenregister 